\title{
Experimental Cryogenic Modeling and Noise of SiGe HBTs
}

\author{
Joseph C. Bardin and Sander Weinreb \\ California Institute of Technology, Pasadena, CA, 91125, USA
}

\begin{abstract}
SiGe devices are an exciting contender for extremely low noise, cryogenically cooled amplifiers. This paper begins with a procedure for extracting a simple equivalent circuit model capable of accurately describing SiGe HBT devices. Next, small-signal modeling results obtained for a $3 \times 0.12 \times 18 \mathrm{um}^{2} \mathrm{SiGe}$ $\mathrm{HBT}$ at 15, 40,77, 120, 200, and $300 \mathrm{~K}$ are presented along with discussion of performance enhancements due to cooling of the device. Finally, the modeled noise performance is presented as a function of temperature and frequency using the concept of minimum cascaded noise temperature, a figure of merit which incorporates both noise temperature and gain.
\end{abstract}

Index Terms - Silicon-germanium (SiGe), cryogenic, low noise amplifier (LNA), noise parameters, transistor modeling.

\section{INTRODUCTION}

Very low-noise amplifiers are critical in systems looking at sources much colder than $300 \mathrm{~K}$. Over the past two decades, the world of extremely low-noise amplification has been dominated by exotic technologies such as InP and GaAs HEMTs [1]. By cryogenically cooling these devices, it is possible to realize microwave amplifiers with noise temperatures as low as $5 \mathrm{~K}$ over decade bandwidths [2]. Although HEMTs can provide very low-noise amplification when cooled to cryogenic temperatures, their radiometer performance is limited by intrinsic transconductance fluctuations [3]. It is believed that bipolar devices do not suffer from this problem.

As industry has invested more and more money in silicon based technologies, SiGe HBT devices have continued to improve and are now at the point where they are beginning to become competitive with InP HEMTs for microwave cryogenic low noise amplifiers [6]. Although extremely high frequency device operation has been observed at cryogenic temperatures [5], little work has been done on modeling the noise of cooled SiGe HBTs (the noise parameters were measured at $85 \mathrm{~K}$ [6]). As a first step at evaluating SiGe HBT devices for use in very low-noise cryogenic amplifiers, a device was modeled at frequencies in the low $\mathrm{GHz}$ range using DC measurements along with room temperature capacitance values and an amplifier was designed which demonstrated a noise temperature of less than $4 \mathrm{~K}$ over 0.5 $3 \mathrm{GHz}$ at $15 \mathrm{~K}$ physical temperature [4].

An outline of the paper is as follows:

1) The small-signal model and a description of the extraction procedure.

2) A comparison of modeled and measured small-signal device performance.

3) The modeled noise performance of the device.

\section{Model EXTRACTION Procedure}

A small-signal equivalent-circuit for the HBT is shown in Fig. 1. While this model is simpler than many models published recently [7], [8], it is believed that it offers sufficient accuracy for modeling transistors of area appropriate to low-noise microwave amplifiers with generators in the $50 \mathrm{ohm}$ range. Furthermore, extraction of base to collector capacitance splitting and other higher order effects is error-prone when the circuit resistances are on the order of just a few ohms, as is the case with the devices investigated in this paper.

In order to determine the parameters across a broad range of biases and temperatures, a combination of DC and RF measurements were made at 15, 40, 77, 120, 200, and 300K. It is assumed that the emitter and substrate are tied to ground. The extraction procedure consists of three steps:

1) Determine $r_{c}, r_{e}, \beta, I_{C}, I_{B}$, and $g_{b e, \text { low_bias }}$ through DC measurements.

2) Determine $C_{c b}$ and $C_{c s}$ through off bias S-parameter measurements.

3) Determine $g_{m}, g_{b e, \text { high_bias }}, r_{b}=r_{b i}+r_{b x}, \tau_{d}$, and $C_{b e}$ through active bias S-parameter measurements.

\section{A. DC Measurements}

A simplified DC equivalent HBT circuit model including bias independent resistances appears in Fig. 2. The first parameter to be extracted is the emitter resistance, $r_{e}$. It is quite important that this resistance is known accurately as it degenerates the transistor, reducing the intrinsic base to emitter voltage for a given base voltage. Therefore, it is measured with two different methods and the results are compared to check for measurement error. The first method employed is the open collector method described in [9]. In this method, the collector is open circuited and the collector voltage is measured as a function of base current. Under such bias conditions, it can be shown that $\partial V_{C} / \partial I_{B} \approx r_{e}$. In addition, it can also be shown that if we allow a forced collector

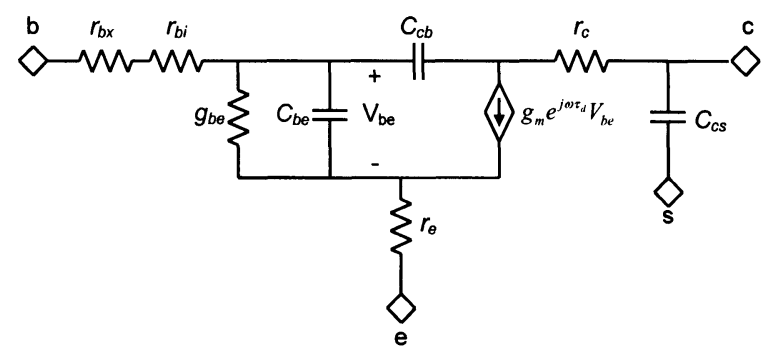

Fig. 1: Small-signal equivalent circuit model. 
current, we can determine the collector resistance: $r_{c} \approx \partial V_{C} / \partial I_{C}-\partial V_{C} / \partial I_{B}$ [9]. It should be noted that the measured collector resistance includes any resistance between the collector current source and the device, so a careful calibration of instrumental resistances is required.

A second method to determine the emitter resistance is through forward Gummel measurements [10]. At high biases, one can easily show that $\partial V_{B} / \partial I_{E} \approx n_{C F} V_{T} / I_{E}+r_{e}$. Therefore, $r_{e}$ can be determined as the y-intercept of a line fit to $\partial V_{B} / \partial I_{E}$ as a function of $1 / I_{E}$. With $r_{e}$ known, $V_{B E}$ can be calculated as a function of $V_{B}, I_{B}$, and $I_{C}$. Forward Gummel measurements are also used to determine the DC I-V characteristics of the device, including $\beta, I_{C}$, and $I_{B}$ as a function of $V_{B E}$ and $V_{C B}$, as well as $g_{b e}=\partial I_{B} / \partial V_{B E}$ and $g_{m}=\partial I_{C} / \partial V_{B E}$. Comparison of transconductance measured in this manner with that calculated using active-bias S-parameters provides a useful check.

\section{B. Off-Bias S-Parameter Measurements}

$C_{c b}$ and $C_{c s}$ are extracted by biasing the device such that all junctions are strongly reverse biased $\left(V_{B E}=0, V_{C}\right.$ swept such that all junctions are reverse biased). In this case, $g_{m}$ and $g_{b e}$ should go to zero, resulting in a device that looks capacitive. From these measurements, we can obtain $C_{c b}$ and $C_{c s}$ :

$$
C_{c b}=\frac{1}{\omega}\left[\frac{1}{\operatorname{Im}\left\{1 / Y_{11}+1 / Y_{12}\right\}}-\frac{1}{\operatorname{Im}\left\{1 / Y_{11}\right\}}\right]
$$

and

$$
\left.C_{c s} \approx \frac{1}{\omega} \operatorname{Im}\left\{Y_{12}+Y_{22}\right\}\right|_{L F}
$$

where LF stands for low frequency.

\section{Active-Bias S-Parameter Measurements}

The remaining parameters are determined through active bias measurements after removing $r_{c}, r_{e}$, and $C_{c s}$ from the measured S-parameters. Measurements are made at a variety of bias points to span a wide operating range. To find $r_{b}, C_{b e}$, and $g_{b e}$, the measured S-parameters are used to calculate the input impedance with the output terminal short circuited:

$$
\frac{1}{Y_{11}}=r_{b}+\frac{g_{b e}-j \omega\left(C_{c b}+C_{b e}\right)}{g_{b e}^{2}+\omega^{2}\left(C_{c b}+C_{b e}\right)^{2}}
$$

For non-zero $g_{b e}$, the imaginary part of (3) has a minima at

$$
\omega_{\min }=\frac{g_{b e}}{C_{b e}+C_{c b}} .
$$

For high biases, $\omega_{\min }$ is in the measurement range and can be used to determine $g_{b e}$ :

$$
g_{b e}=-\frac{1}{2 \operatorname{Im}\left\{1 / Y_{11}\left(\omega_{\min }\right)\right\}} .
$$

Prior to determining $\omega_{\min }$ it may be desirable to fit a smooth curve to the imaginary component of the short-circuit input impedance to avoid underestimating $g_{b e}$ due to measurement noise. For the low bias case, $g_{b e}$ must be found using the forward Gummel method. $C_{b e}$ is calculated as

$$
C_{b e}=-\frac{1}{\omega \Im\left\{1 / Y_{11}\right\}}-C_{c b}
$$

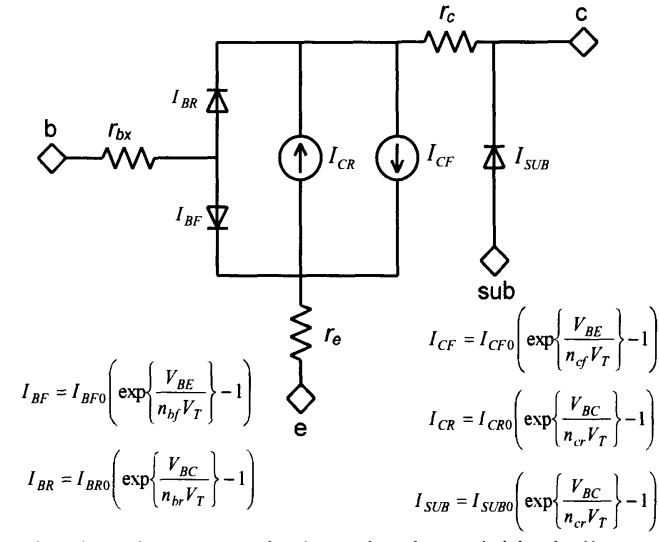

Fig 2: Simple DC HBT equivalent circuit model including access resistances.

With $C_{c b}, C_{b e}$, and $g_{b e}$ all known, the base resistance can now be calculated by evaluating the real part of (3) at high frequencies. The final two parameters, $g_{m}$ and $\tau_{d}$ can be obtained using the procedure given in [7].

\section{MODEL VERIFICATION AND SMALl Signal PERFoRMANCE}

The procedure described in section II was used to extract equivalent circuit models as a function of temperature and bias for a $3 \times 0.12 \times 18 \mu \mathrm{m}^{2}$ IBM BiCMOS 8 HP HBT on IBM multiproject wafer KCPTF12, received in December, 2006. Using a custom-built cryogenic wafer-probe station, on-wafer measurements were made at $15,40,77,120,200$, and $300 \mathrm{~K}$. At each temperature, a SOLT calibration was performed. On wafer open and short standards were then used to remove the effects of pad capacitance and the transistor feedlines. Unfortunately the structures do not remove the effects of wiring very close to the device, leading to higher capacitance measurements than are typical of this technology.

Following measurements, the data were post-processed in Matlab and the small-signal parameters were extracted. Model parameters for the HBT biased for peak $\beta$ and for $J_{C}=1 \mathrm{~mA} / \mu \mathrm{m}^{2}$ appear in tables I and II respectively. A graphical comparison of modeled and measured data between 0.3 and $40 \mathrm{GHz}$ with the transistor operating at $15 \mathrm{~K}$ is shown in fig. 3. These plots are representative of typical results achieved, confirming that the modeling procedure provides an excellent fit to the data over a wide range of biases. Fig. 4 shows both measured $\beta$ and measured unity current gain frequency, $f_{t}$, as a function of $J_{C}$. It is interesting to note that the peaks occur at different collector current densities.

Table II provides useful insight into the performance improvement that comes with cooling the device. For example, looking at $f_{t}$, it can be seen that for a given collector current density, faster device operation is realized at cryogenic temperatures than under normal operating conditions (of course, this assumes that the collector current density is low enough that $f_{t}$ has not begun rolling off due to the Kirk effect [11]). This is not surprising as the transconductance has increased by a factor of around three and a half from its room 
temperature value by the time the device reaches $15 \mathrm{~K}$. However, the increase in $f_{t}$ is not as large as the increase in transconductance because the base to emitter capacitance has increased by a factor of about two. It is believed that this increase in capacitance is due to the increase in the device turn on voltage from $\sim 0.65 \mathrm{~V}$ at room temperature to $\sim 1 \mathrm{~V}$ at $15 \mathrm{~K}$.

\section{NOISE PERFORMANCE}

The noise parameters of a SiGe HBT device can be determined at a given bias from its small signal e uivalent circuit along with a delay term which determines the correlation between the input and output shot noise generators [12]. This delay term was not measured and will be ignored for the following analysis resulting in a possible overestimation of noise at high fre uencies.

The optimization of the noise performance of a device re uires consideration of both the noise figure, $F$, and the exchangeable gain, $G_{e}$ similar to available gain, but extended to devices which are potentially unstable [13]). A figure of merit which takes both of these properties into account is the noise measure, $M$, of the device. The noise measure is the noise temperature of an infinite cascade of devices, $T_{C A S}$, normalized to $T_{o}=290 \mathrm{~K}$. Thus the cascaded noise $p r \quad r, T_{C A S}$ is given by,

$$
T_{C A S}=T_{0} M=T_{0} \frac{F-1}{1-1 / G_{e}}=\frac{T_{n}}{1-1 / G_{e}} .
$$

For large gain $T_{C A S}$ is e ual to the noise temperature, $T_{n}$, of the device, but for low gain, at high fre uencies, it is higher due to the contribution of following stages.

The cascaded noise temperature, $T_{C A S}$, of a device has a very fundamental property that its minimum value with respect to

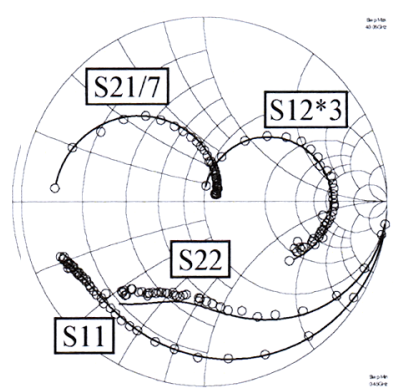

(a)

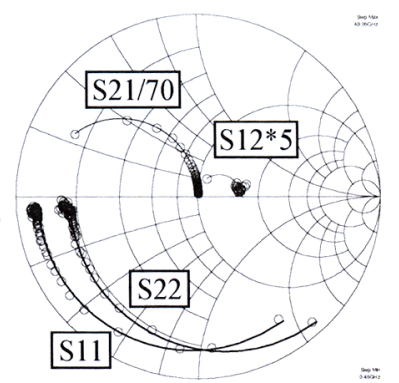

(b)
Fig 3: Modeled (solid) and measured (circles) S-Parameters at $\mathrm{T}=15 \mathrm{~K}$. (a) Biased for peak $\beta$. (b) Biased for $J_{C}=1 \mathrm{~mA} / \mu \mathrm{m}^{2}$.

source impedance, $T_{C A S, M I N}$, is invariant to any lossless network that embeds the device; that is, it is invariant to lossless input, output, and feedback networks [13]. A lossy or noisy network can only increase $T_{C A S, M I N}$.

$T_{C A S, M I N}$ can be computed using the relations for minimum noise measure in terms of the measured e uivalent circuit parameters as a function of DC bias, the DC currents which determine shot noise magnitudes, and physical temperature which determines the thermal noise [ ],[13]-[16]. Fig. 5 shows the results of this computation with the additional constraint that $T_{C A S, M I N}$ is also minimized with respect to collector current density, $J_{C}$, at each fre uency. The optimum current density and the optimum source impedance for a $3 \times 0.12 \times 1 \mathrm{um}^{2}$ device also appear in fig. 5 .

As the device is cooled from $300 \mathrm{~K}$, the minimum value of $T_{C A S, M I N} J_{C}$ ) decreases by a factor of two at $200 \mathrm{~K}$, a factor of eight at $77 \mathrm{~K}$, and a factor of fifteen at $15 \mathrm{~K}$. These are exciting results because, in addition to the very low noise performance at $15 \mathrm{~K}$, they demonstrate that the noise performance can be

TABLE I

Summary OF EXTRACTED PARAMETER VALUES AT PEAK $\beta, \mathrm{VCB}=0.5 \mathrm{~V}$

\begin{tabular}{|l|l|l|l|l|l|l|l|l|l|l|l|l|}
\hline $\mathrm{T}$ & $r_{e}$ & $r_{b}$ & $r_{c}$ & $g_{m}$ & $g_{b e}$ & $C_{c b}$ & $C_{b e}$ & $C_{c s}$ & $\tau_{d}$ & $\beta$ & $f_{t}$ & $J_{C}$ \\
\hline $\mathrm{K}$ & $\Omega$ & $\Omega$ & $\Omega$ & $\mathrm{mS}$ & $\mu \mathrm{S}$ & $\mathrm{fF}$ & $\mathrm{fF}$ & $\mathrm{fF}$ & $\mathrm{pS}$ & - & $\mathrm{GHz}$ & $\mathrm{mA} / \mu \mathrm{m}^{2}$ \\
\hline 15 & 0.5 & 1.0 & 0.3 & 60 & 5.9 & 97 & 272 & 75 & 0.6 & 8400 & 26 & 0.07 \\
\hline 40 & 0.5 & 1.6 & 0.2 & 107 & 12.9 & 101 & 284 & 60 & 0.9 & 7400 & 44 & 0.14 \\
\hline 77 & 0.5 & 1.2 & 0.4 & 71.7 & 14.7 & 101 & 258 & 69 & 0.7 & 5240 & 32 & 0.12 \\
\hline 120 & 0.5 & 1.0 & 0.7 & 24.7 & 11.2 & 99 & 216 & 70 & 0.7 & 2240 & 13 & 0.05 \\
\hline 200 & 0.6 & 2.2 & 0.9 & 12.3 & 19.9 & 98 & 191 & 70 & 1.0 & 680 & 8 & 0.04 \\
\hline 300 & 0.7 & 1.9 & 1.9 & 8.4 & 25.4 & 96 & 184 & 60 & 0.6 & 320 & 5 & 0.03 \\
\hline
\end{tabular}

TABLE II

SUMMARY OF EXTRACTED PARAMETER VALUES AT $\mathrm{J}_{\mathrm{C}}=1 \mathrm{~mA} / \mu \mathrm{m}^{2}$ AND VCB $=0.5 \mathrm{~V}$

\begin{tabular}{|l|l|l|l|l|l|l|l|l|l|l|l|}
\hline $\mathrm{T}$ & $r_{e}$ & $r_{b}$ & $r_{c}$ & $g_{m}$ & $g_{b e}$ & $C_{c b}$ & $C_{b e}$ & $C_{c s}$ & $\tau_{d}$ & $f_{t}$ & $\beta$ \\
\hline $\mathrm{K}$ & $\Omega$ & $\Omega$ & $\Omega$ & $\mathrm{mS}$ & $\mathrm{mS}$ & $\mathrm{fF}$ & $\mathrm{fF}$ & $\mathrm{fF}$ & $\mathrm{pS}$ & $\mathrm{GHz}$ & - \\
\hline 15 & 0.5 & 1.0 & 0.3 & 804 & 2.0 & 97 & 684 & 75 & 1.42 & 162 & 1400 \\
\hline 40 & 0.5 & 1.6 & 0.2 & 742 & 1.6 & 101 & 659 & 60 & 0.72 & 162 & 1320 \\
\hline 77 & 0.5 & 1.2 & 0.4 & 546 & 1.4 & 101 & 464 & 69 & 0.35 & 146 & 1030 \\
\hline 120 & 0.5 & 1.0 & 0.7 & 394 & 1.4 & 99 & 374 & 70 & 0.74 & 120 & 656 \\
\hline 200 & 0.6 & 1.8 & 0.9 & 260 & 1.1 & 98 & 336 & 70 & 0.45 & 94 & 408 \\
\hline 300 & 0.7 & 1.8 & 1.9 & 224 & 1.1 & 96 & 306 & 60 & 0.17 & 79 & 266 \\
\hline
\end{tabular}

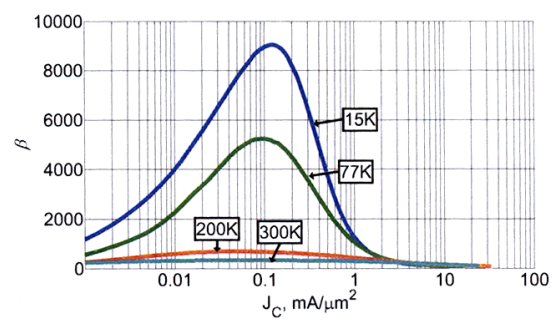

(a)

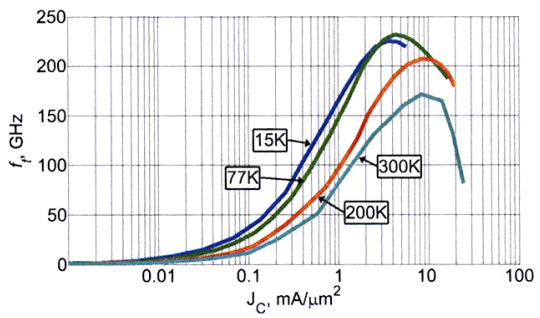

(b)

Fig 4: Measured $\beta$ (a) and $f_{t}(\mathrm{~b})$ as a function of collector current density. $V_{C B}=0.5 \mathrm{~V}$. It is noteworthy that peak $\beta$ and peak $f_{t}$ occur at quite different current densities. 
greatly enhanced using thermoelectric coolers reaching $200 \mathrm{~K}$, and inexpensive refrigerators reaching $77 \mathrm{~K}$.

\section{CONCLUSIONS}

A procedure has been presented and applied to model a $3 \times 0.12 \times 18 \mu \mathrm{m}^{2}$ SiGe HBT at temperatures ranging from 15-300K. Excellent agreement has been demonstrated between modeled and measured data. Following modeling, noise properties of the device were investigated as a function of temperature and it was found that excellent performance can be achieved at cryogenic temperatures. Future areas of research include modeling devices as a function of emitter length, including shot noise correlation in the model, and the design of an amplifier in the tens of $\mathrm{GHz}$ range.

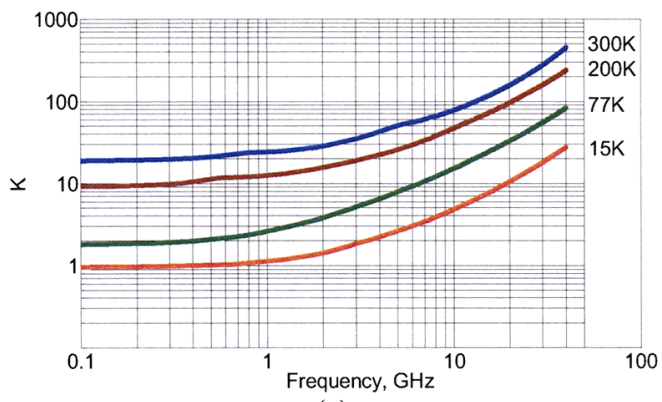

( )

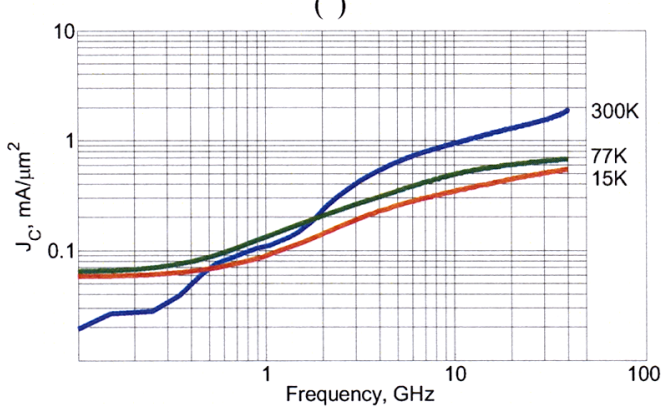

(b)

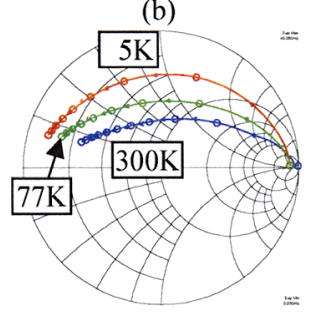

(c)

Fig. 5: (a) Minimum value of $T_{C A S, M I N}\left(J_{C}\right)$ versus frequency for the modeled $3 \times 0.12 \times 18 \mu \mathrm{m}^{2} \mathrm{HBT}$ at $15,77,200$, and $300 \mathrm{~K}$. (b) Modeled optimum value of collector current density to produce the minimum cascaded noise temperature. The trend is towards maximum $\beta$ bias at low frequencies and towards higher $f_{t}$ bias at high frequencies. Some perturbations in the curves are due to the shallow minima. A current density of $1 \mathrm{~mA} / \mu \mathrm{m}^{2}$ corresponds to $6.5 \mathrm{~mA}$ collector current in a $3 \times 0.12 \times 18 \mu \mathrm{m}^{2}$ device. (c) Optimum source impedance required to achieve cascaded noise temperature shown in (a). Frequency range is $0.05-40.5 \mathrm{GHz}$ with markers spaced every $5 \mathrm{GHz}$. $V_{C B}=0.5 \mathrm{~V}$ for all three plots.

\section{ACKNOWLEDGEMENT}

We appreciate the help of the Caltech Mixed Signal and RF Group, particularly, Prof. Ali Hajimiri, Aydin Babakhani, Florian Bohn, Hua Wang, and Yu-Jiu Wang for help with the IBM process. We thank Prof. David Rutledge and Glenn Jones for helpful discussions of the work, Hamdi Mani for help with assembly, and Ezra Long, Javier Bautista, and Jim Shell for measurement assistance. The tested devices were processed by IBM under the Trusted Foundry Program with sponsorship of Dr. K. Johnson of the US Naval Observatory. This work was supported by the Director's Fund of the Jet Propulsion Laboratory, California Institute of Technology, under contract with the National Aeronautics and Space Agency.

\section{REFERENCES}

[1] M. Pospieszalski, "Extremely low-noise amplification with cryogenic FETs and HFETs: 1970-2004," IEEE Microwave Magazine, Vol. 6, pp.72-75, Sept. 2005.

[2] J. Pandian et al., "Low-noise 6-8GHz Receiver," IEEE Microwave. Magazine, Vol. 7, pp.74-84, Dec. 2006.

[3] N.C. Jarosik, "Measurements of the low-frequency-fluctuations of a $30-\mathrm{GHz}$ high-electron-mobility-transistor cryogenic amplifier," IEEE Trans. MTT, Vol. 44, pp193-197, Feb 1996.

[4] S. Weinreb, J.C. Bardin, H. Mani, "Design of cryogenic SiGe low noise amplifiers," IEEE Trans. MTT, Vol. 55, pp2306-2312, Nov. 2007.

[5] J. Yuan et al., "On the frequency limits of SiGe HBTs for TeraHertz applications," Proc. IEEE BCTM, pp.22-25, Sept. 2007.

[6] B. Banerjee et al., "Broadband noise modeling of SiGe HBT under cryogenic temperatures," Proc. RFIC pp. 765-768, June 2007.

[7] T. Yang et al., "SiGe HBT's small-signal Pi modeling," IEEE Trans. MTT, Vol. 55, pp.1417-1424, July 2007.

[8] K. Xia, Improved RF Noise Modeling for Silicon Germanium Heterojunction Bipolar Transistors, Auburn University: PhD Dissertation, Dec. 2006.

[9] M. Rudolph, Introduction to Modeling HBTs, Massachusetts: Artech House, INC: 2006.

[10] R. Anholt, Electrical and Thermal Characterization of MESFETS, HEMTs, and HBTs, Massachusetts: Artech House INC: 1995.

[11] C.T. Kirk Jr., "A theory of transistor cutoff frequency $\left(f_{t}\right)$ falloff at high current densities," IEEE Trans. on Elec. Dev., Vol. 9, pp.164-174, Mar. 1962.

[12] G. Niu et al., "A unified approach to RF and microwave noise parameter modeling in bipolar transistors," IEEE Trans. Elec. Dev., Vol. 48, pp.2568-2574, Nov. 2001.

[13] H. A. Haus and R. B. Adler, "Optimum noise performance of linear amplifiers," Proc. IRE, Vol. 46, pp. 1517-1533, Aug. 1958.

[14] J. D. Cressler and G. Niu, Silicon Germanium Heterojunction Bipolar Transistors, Massachusetts: Artech House, 2003.

[15] H. Fukui, "Available power gain, noise figure, and noise measure of two-ports and their graphical representations," IEEE Trans. Circuit Theory, Vol. 13, pp.137-142, June 1966.

[16] C. R. Poole and D. K. Paul, "Optimum noise measure terminations for microwave transistor amplifiers," IEEE Trans. MTT, Vol. 33, pp. 1254-1257, Nov 1985. 